\title{
LETTER
}

\section{Color Constancy Based on Effective Regions}

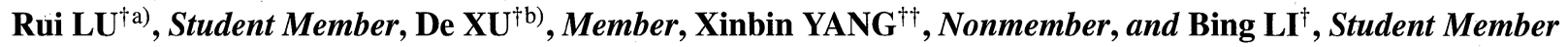

SUMMARY None of the existing color constancy algorithms can be considered universal. Furthermore, they use all the image pixels, although actually not all of the pixels are effective in illumination estimation. Consequently, how to select a proper color constancy algorithm from existing algorithms and how to select effective(or useful) pixels from an image are two most important problems for natural images color constancy. In this paper, a novel Color Constancy method using Effective Regions (CCER) is proposed, which consists of the proper algorithm selection and effective regions selection. For a given image, the most proper algorithm is selected according to its Weilbull distribution while its effective regions are chosen based on image similarity. The experiments show promising results compared with the state-of-the-art methods.

key words: color constancy, illumination estimation, image similarity, Weilbull distribution

\section{Introduction}

The color of an object recorded by electronic devices is subject to the color of the light source. As a result, the same object viewed under different illuminations may appear different colors. It is biased towards the color of the light source, which will bring us a lot of trouble in reality. Color constancy aims at getting object color independent of illuminations. It can be applied to many fields of image processing and computer vision such as image segmentation, scene understanding and object recognition [1].

A lot of methods on color constancy have been proposed until now. They can be roughly divided into two main groups: statistical-based and physics-based approaches [2]. Statistical-based approaches try to estimate the scene illumination with statistical knowledge of common lights and surfaces: for example, Gray-World algorithm assumes that the average reflectance of the image is achromatic. White Patch algorithm assumes that the maximum response in a scene is caused by a perfect reflectance [2]. By contrast, physicsbased approaches try to estimate the scene illumination with knowledge about the physical interaction between light and surfaces.

Since all of the existing color constancy algorithms are based on some assumptions, none of them can be considered as universal. For example, White-Patch algorithm per-

\footnotetext{
Manuscript received December 18, 2007.

Manuscript revised March 31, 2008.

${ }^{\dagger}$ The authors are with Institute of Computer Science and Engineering, Beijing Jiaotong University, Beijing, China.

${ }^{\dagger \dagger}$ The author is with Dongying Vocational College, Shandong, China.

a) E-mail: lurui1983@126.com

b)E-mail: dxu@bjtu.edu.cn

DOI: 10.1093/ietisy/e91-d.7.2091
}

forms best on images with many textures while the general Grey-World algorithm performs better on images with less texture and contrast. Therefore, how to combine them is one of the challenging but promising research directions of this field [2].

Most of the existing algorithms use all the pixels in a given image. As a matter of fact, not all of the pixels in the image are useful in illumination estimation. For example, in the image shown in Fig. 1, blue sky covers nearly a half of the total image. Pixels in this area will cause illumination estimation to bias blue. So it is reasonable to discard some pixels in this area.

In this paper a novel color constancy algorithm based on effective regions (CCER) is proposed. In this method the most proper algorithm is selected among five different color constancy algorithms first. Then the effective regions for an image is chosen based on image similarity. Finally, the color of the light source is estimated using the effective regions rather than the whole image.

The remainder of this paper is organized as follows: Section 2 briefly describes the color constancy problem. In Sect. 3, we discuss the proposed algorithm in detail. The experimental results are shown in Sect. 4. Subsequently the conclusion is drawn in Sect. 5.

\section{Color Constancy}

According to the Lambertian reflectance model, the image $f=(R, G, B)^{T}$ recorded by electronic devices is the product of the object reflectance properties and the illumination spectrum, i.e.

$$
f(x)=\int_{w} e(\lambda) s(x, \lambda) c(\lambda) d \lambda
$$

where $x$ is spatial coordinate, $\lambda$ is wavelength and $w$ represents visible spectrum. $e(\lambda)$ is color of light source, the surface reflectance is denoted as $s(x, \lambda)$ and the camera sensitivity function $c(\lambda)=(R(\lambda), G(\lambda), B(\lambda))$. Color constancy aims at estimating $e$ :

$$
e=\int_{w} e(\lambda) c(\lambda) d \lambda
$$

Obviously, color constancy is an inherently underconstrained problem.

To solve this problem, several assumptions have been proposed. More recently, a new unified color constancy 
framework was proposed, which is based on the assumption that the average reflectance of edges in the scene is achromatic [3]:

$$
\left(\int\left|\frac{\partial^{n} f^{\sigma}(x)}{\partial x^{n}}\right|^{p}\right)^{\frac{1}{p}}=k e^{n, p, \sigma}
$$

where $n$ is the order of the derivative, $p$ is the Minkowski norm and $f^{\sigma}=f \otimes G^{\sigma}$ is the convolution of the image with a Gaussian filter with scale parameter $\sigma$. Many color constancy algorithms can be obtained by changing the value of the parameters in Eq. (3). In our experiments, for the ease of illustration and comparison, we focus on five candidate algorithms: $e^{0,1,0}$ (Grey-world algorithm), $e^{0, \infty, 0}$ (White-Patch algorithm), $e^{0,13,2}$ (General Grey-World algorithm), $e^{0,1,6}$ (first-order Grey-Edge algorithm) and $e^{2,1,5}$ (second-order Grey-Edge algorithm).

\section{Color Constancy Based on Effective Image Regions}

\subsection{Algorithm Description}

Most of the existing algorithms use all of the pixels in the image. Actually this is not reasonable, because some of the pixels may cause the estimation biased. For example, if Grey-World algorithm is used, illuminations estimation will be biased toward the prevailing color of the image. The proposed algorithm, which uses effective regions, is inspired by this fact.

The proposed algorithm is carried out in training phase and test phase two steps.

\section{Training phase}

Step 1. For each image, the color of the light source is estimated using the five color constancy algorithms mentioned above separately. And then the results are compared with the ground truth. The algorithm with the smallest angular error(see Eq. (7) for details) is selected, denoted as $A$, which is considered as the optimal color constancy algorithm for this image. From now on we will just use algorithm $A$ to estimate the illumination of this image.

Step 2. The image is segmented into regions. For each region we will estimate the color of the light source using algorithm $A$. Regions with the smallest error are selected and considered as the effective regions of this image. See Sect. 3.2 for details about effective regions selection.

\section{Testing phase}

For a given test image $I_{t}$, search an image $I_{r}$ which is the most similar to $I_{t}$ in the training data set. Please refer to Sect. 3.3 for details in image similarity measurement. Then provided that $I_{r}$ has an effective region $R_{r}$ and an optimal algorithm $A_{r}$, we will use $A_{r}$ to estimate the illumination of $R_{t}$ of $I_{t}$, where $R_{t}$ is at the same location as $R_{r}$ of $I_{r}$. The illumination estimation of $R_{t}$ is served as the illumination estimation of $I_{t}$.

In conclusion, for each image in the training data set, the most proper algorithm and its corresponding effective regions are selected. If images in the training data set are changed, then proper algorithms and their corresponding effective regions must be recomputed. For each test image, we use the same algorithm and effective regions as the image in the training data set, which is the most similar to it.

\subsection{Effective Image Regions Selection}

Recently, Gijsenij et. al. put forward color constancy algorithm using image regions (CCIR) [4]. Their approach is quite novel, but there still exist several points to improve. First, they just use a specific color constancy algorithm, i.e. max-RGB. Maybe it is not the best choice for every image. Second, their algorithm is based on the assumption that all the images have the same useful regions if they belong to the same category. Obviously, it doesn't make sense under any conditions, and to get useful regions for a given category, quite a lot of training images are needed.

Consequently, a novel algorithm using effective regions based on image similarity is proposed in this paper. In this algorithm effective image regions are obtained based on image similarity to get rid of scene category limitations. Furthermore, the effective regions are associated with the best color constancy algorithm chosen from five different color constancy algorithms.

The effective regions selection process is as follows. For each image in the learning data set:

- Step 1. The color of the light source is estimated using each of the five algorithms mentioned above. The results are compared with the ground truth.

- Step 2. The algorithm with the smallest angular error is selected and denoted as $A$.

- Step 3. The image is segmented into regions. To make the segmentation simple, a regular grid segmentation is used in our algorithm, as is shown in Fig. 1(B). Other segmentation algorithm may be used as well.

- Step 4. For each region, the color of the light source is estimated using algorithm $A$. Then the results are compared with the ground truth. Thus, we can get an angular error for each region $\varepsilon_{i}, i \leq n u m$, where num is the number of the regions.

- Step 5. The angular errors $\varepsilon$ are ranked from low to high. At first, the region with the smallest error is considered as the effective region Re. Next, the region with second smallest error will be incorporated into Re to form a new effective region Renew. Then we will estimate the color of the light source over the new effective region Renew and compare the estimation with the ground truth. If the new error $\varepsilon_{\text {new }}$ is bigger than the one before, we will stop and consider Re is the final effective region of this image. Otherwise, we will continue to incorporate the region with the next smallest error and repeat above processing.

In this way effective regions are obtained for each training image, as shown in Fig. 1 (C). 


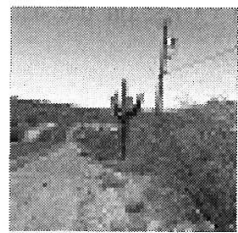

(A) Original

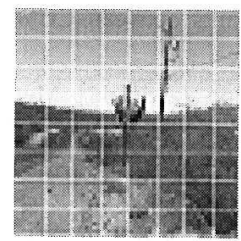

(B) Segmentation

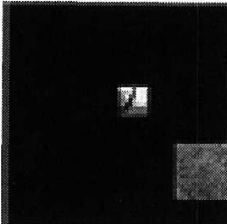

(C) Effective Regions
Fig. 1 An example. For a given image, we first segment the image into a $8 \times 8$ grid, then estimate illumination for each region. Finally, the effective regions with smaller angular error are obtained.

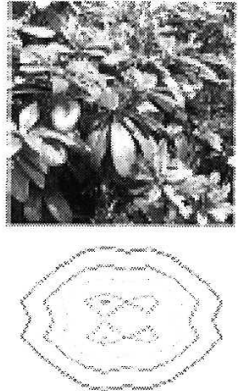

(A)
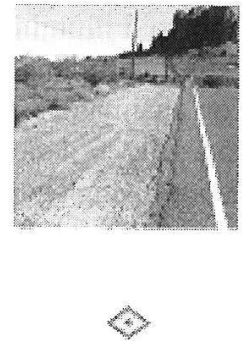

(B)
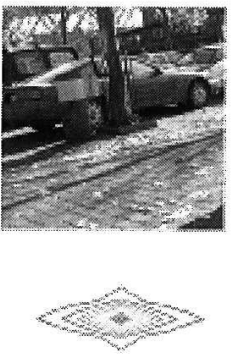

(C)
Fig. 2 Relationship between spatial texture and Weilbull distribution.

\subsection{Image Similarity Computation}

Spatial structure is a valuable feature for natural images. Weibull distribution can characterize the spatial structure of uniform stochastic texture of image [5], as is shown in Fig. 2. It's demonstrated that images with same spatial structure may choose same color constancy algorithms [6]. Therefore, image similarity is computed based on Weibul distribution.

Edge responses $f(x)$ of an image can be modelled through Weilbull distribution [6].

$$
f(x)=\frac{\gamma}{\beta}\left(\frac{x}{\beta}\right)^{\gamma-1} e^{-\left(\frac{x}{\beta}\right)^{\gamma}}
$$

where $\beta$ is the width of the distribution, which means the contrast of the image, while $\gamma$ is the peakedness of the distribution, which means the grain size of the image. That means, a smaller $\beta$ value indicates less contrast, while a smaller $\gamma$ value indicates a bigger grain size (more rough textures). Figure 2 clearly demonstrates that differences in contrast and grain size of images lead to great differences in Weilbull distribution.

In our algorithm, edge responses are computed using a Guassian derivative filter in order to get the Weilbull distribution. In [6], it is shown that spatial statistics can be got through a single filter type. In this paper, a first-order derivative filter in the $x$ and $y$-direction is used. There are great differences among Weilbull parameters of different parts of natural images. An image can roughly be divided into topdown structure or left-right structure. Hence, to thoroughly exploit image statistics knowledge, we split the image into top-bottom at the horizontal center and left-right at the vertical center, respectively. Then Weilbull parameters are computed along $\mathrm{x}$ and $\mathrm{y}$-direction for each part of the image, resulting in a feature vector $F$ with ten $\beta$ and ten $\gamma$. That is to say,

$$
F=\left[f_{\text {top }}, f_{\text {down },}, f_{\text {left },} f_{\text {right },}, f_{\text {whole }}\right]
$$

where $f=\left(\beta_{x}, \gamma_{x}, \beta_{y}, \gamma_{y}\right) . f$ is obtained through image top half part, bottom half part, left half part, right half part and the whole image, individually.

Given two images $I_{i}$ and $I_{j}$, their corresponding feature vectors are $F_{i}$ and $F_{j}$, their similarity are computed as follows:

$$
\operatorname{Dist}\left(F_{i,} F_{j}\right)=\sum_{k=1}^{20}\left(\hat{F}_{i}(k)-\hat{F}_{j}(k)\right)^{2}
$$

where denotes the $k^{\text {th }}$ component of the normalized vector of $F_{i}$. The smaller $\operatorname{Dist}\left(F_{i} F_{j}\right)$ is, the more similar the two images are. And so there is great possibility that we can use the same color constancy algorithm based on similar image regions.

\section{Experiments}

Data set: The proposed color constancy algorithm is evaluated on a large data set [7], including more than 11,000 images. All of the images extracted from 15 different video clips cover different kind of scenes. Note that our experiments use the modified images with the gray ball masked.

\subsection{Performance Measurement}

For a given image $I$ with its estimated illumination $e_{e}=\left(R_{e}, G_{e}, B_{e}\right)^{T}$ and its measured illumination $e_{l}=$ $\left(R_{l}, G_{l}, B_{l}\right)^{T}$, the angular error is used to measure algorithm performance.

$$
\varepsilon=\cos ^{-1}\left(\hat{e}_{e} \cdot \hat{e}_{l}\right)
$$

where $\hat{e}_{e} \cdot \hat{e}_{l}$ is the dot product of the two normalized vectors of $e_{e}$ and $e_{l}$. In order to measure our algorithm more objectively, median and mean angular error on the whole test set are utilized in our algorithm [8].

\subsection{Experiment Results}

We firstly use the same images in our experiment as CCIR [4]. For the ease of comparison, we also classify the images used in the experiment into three categories: Open Country, Street and Indoor. The comparison results are shown in Table 1.

In the following, we compare the proposed algorithm with the leading whole-image based color constancy algorithms using data set [7]. In the experiment, the data set is divided into three groups. In algorithm [6], they use two groups of the three as training data set while the rest is used 
Table 1 Comparison results with region-based color constancy algorithm [4].

\begin{tabular}{|l|c|c|c|c|}
\hline \multirow{2}{*}{} & \multicolumn{2}{|c|}{ Mean } & \multicolumn{2}{c|}{ Median } \\
\cline { 2 - 5 } & CCIR & CCER & CCIR & CCER \\
\hline Open Country & $6.0^{\circ}$ & $4.9^{\circ}$ & $6.2^{\circ}$ & $3.5^{\circ}$ \\
\hline Street & $4.9^{\circ}$ & $2.6^{\circ}$ & $3.5^{\circ}$ & $1.6^{\circ}$ \\
\hline Indoor & $4.7^{\circ}$ & $2.4^{\circ}$ & $4.0^{\circ}$ & $2.0^{\circ}$ \\
\hline
\end{tabular}

Table 2 Comparison results on the data set [7] with whole-image based color constancy algorithms.

\begin{tabular}{|l|c|c|}
\hline Method & Mean & Median \\
\hline (1)Grey-World & $7.9^{\circ}$ & $7.0^{\circ}$ \\
\hline (2)White-Patch & $6.8^{\circ}$ & $5.3^{\circ}$ \\
\hline (3)General Grey-World & $6.2^{\circ}$ & $5.3^{\circ}$ \\
\hline (4)1st-order Grey-Edge & $6.2^{\circ}$ & $5.2^{\circ}$ \\
\hline (5)2nd-order Grey-Edge & $6.1^{o}$ & $5.2^{\circ}$ \\
\hline (6)Selection(5 methods) & $5.7^{\circ}$ & $4.7^{\circ}$ \\
\hline (7)Combination(5 methods) & $5.6^{\circ}$ & $4.6^{\circ}$ \\
\hline (8)Combination(75 methods) & $5.0^{\circ}$ & $3.7^{\circ}$ \\
\hline (9)CCER & $4.7^{\circ}$ & $3.3^{\circ}$ \\
\hline
\end{tabular}

as test data set. We use only one group of the three as training data set while one of the other two is test data set. All six permutations of the three groups are tested. The average experimental results are shown in Item(9) of Table 2. Methods (6) (7) and (8) are proposed in [6]. Their corresponding results are taken directly from [6]. According to [6], their algorithm outperforms all the algorithms available at that time. Table 2 clearly demonstrates that our algorithm is competitive with theirs. Provided that the five candidate methods mentioned above are concerned, mean error is reduced by $16 \%$ while median error is reduced by $28 \%$ compared with their method. Note our training data set is only a half of the training data set used in [6] while test data set is the same. Even so, our algorithm still outperforms theirs.

To further convince that the proposed idea using effective regions is better than using the whole image, another experiment using the data set [7] is carried out. The experiment compares two algorithms: Color Constancy algorithm based on the Whole Image (CCWI) and Color Constancy algorithm based on Effective Regions (CCER). Both of them use the same proper color constancy algorithm selected from five candidates mentioned above. In this experiment, all of the images in the data set [7] are made full use of. The data set is divided into two parts. One part with only 1800 images extracted with equal space from 11,000 images is used as the training data set while the other part is used as the test data set. The ratio between the two parts amounts to 1:5. The comparison results are shown in Table 3 . From the table, it clearly demonstrates that the method using effective regions outperforms the method using the whole image.
Table 3 Comparison results between CCWI and CCER on the data set [7].

\begin{tabular}{|c|c|c|}
\hline Method & Mean & Median \\
\hline CCWI & $5.9^{\circ}$ & $4.5^{\circ}$ \\
\hline CCER & $5.1^{\circ}$ & $3.8^{\circ}$ \\
\hline
\end{tabular}

\section{Conclusion}

A novel color constancy algorithm based on effective regions(CCER) is proposed in this paper. Unlike most of the algorithms available, it uses effective regions rather than the whole image in order to avoid illumination estimation bias. Meanwhile the best algorithm among the five candidate algorithms is selected for an image, since none of them is universal. Evaluated on a large data set, the results show that CCER can achieve promising results compared with the state-of-the-art algorithms.

\section{Acknowledgement}

This paper is supported by Chinese National Programs for High Technology Research and Development (Project No.2007AA01Z168).

The authors would like to thank Prof. Brian Funt for providing them the large data set with more than $11,000 \mathrm{im}-$ ages. They would also like to thank Mr. Arjan Gijsenij for his valuable advice. They are also grateful to the reviewers. They helped to improve the paper significantly.

\section{References}

[1] K. Barnard, V. Cardei, and B.V. Funt, "A comparison of computational color constancy algorithms-part 1: Methodology and experiments with synthesized data," IEEE Trans. Image Process., vol.11, no.9, pp.972-983, Sept. 2002.

[2] S. Hordley, "Scene illuminant estimation: Past, present, and future," Color Research and Application, vol.31, no.4, pp.303-314, 2006.

[3] J. van de Weijer, T. Gevers, and A. Gijsenij, "Edge-based color constancy," IEEE Trans. Image Process., vol.16, no.9, pp.2207-2214, 2007.

[4] A. Gijsenij and T. Gevers, "Color constancy using image regions," IEEE Conf. on Image Proceeding, vol.3, pp.501-504, Sept. 2007.

[5] J. Geusebroek and A. Smeulders, "A six-stimulus theory for stochastic texture," Int. J. Comput. Vis., vol.62, no.1-2, pp.7-16, 2005.

[6] A. Gijsenij and T. Gevers, "Color constancy using image statistics," IEEE Conf. on Computer Vision and Pattern Recognition, pp.1-8, June 2007.

[7] F. Ciurea and B. Funt, "A large image database for color constancy research," The Eleventh Color Imaging Conference, IS\&T - The Society for Imaging Science and Technology, pp.160-164, 2003.

[8] S. Hordley and G.D. Finlayson, "Reevaluation of color constancy algorithm performance," J. Optical Society of America, vol.23, no.5, pp.1008-1020, 2006. 